\title{
Application of a Montecarlo based quantitative Kramers-Kronig test for linearity assessment of EIS measurements
}

\author{
J. J. Giner-Sanz, E. M. Ortega, V. Pérez-Herranz* \\ IEC group, Depto. Ingeniería Química y Nuclear, Universitat Politècnica de València \\ Camino de Vera S/N, 46022 Valencia, Spain \\ *Corresponding author. Tel.: +34-96-3877632; fax: +34-96-3877639; \\ E-mail address: vperez@iqn.upv.es (V.Pérez-Herranz)
}

\section{Abstract:}

Electrochemical Impedance Spectroscopy (EIS) is a very powerful tool to study the behaviour of electrochemical systems. Three conditions must be fulfilled during EIS measurements: causality, linearity, and stability. If any of these conditions is not achieved, then the conclusions obtained from the analysis of the measured spectra may be biased, or even misguided. For this reason, the verification of the compliance of these conditions is critical before accepting any analysis performed on an experimental spectrum. In a previous work, an experimental spectrum quantitative validation technique based on Kramers-Kronig relations was presented. The validation method consists in a Kramers-Kronig (KK) validation test, by equivalent electrical circuit fitting, coupled with a Montecarlo error propagation method. The validation technique builds a consistency region for a given confidence level that allows to discriminate between the individual points of the EIS spectrum that are consistent with the four fundamental hypotheses, and the inconsistent ones. The aim of this work is to validate experimentally if the quantitative validation technique is able to detect nonlinearities. In order to achieve this goal, the EIS spectrum of a markedly nonlinear system was measured experimentally using different perturbation amplitudes. The validation technique was applied to each one of the measured spectra. The method successfully managed to identify large nonlinearities; but did not detect slight ones.

Keywords: Electrochemical impedance spectroscopy (EIS), Kramers-Kronig, Montecarlo algorithm, Quantitative validation, Linearity assessment method. 


\section{Introduction}

The Electrochemical Impedance Spectroscopy (EIS) is an electrochemical technique that consists in the application of a sinusoidal voltage or current signal (perturbation signal) of known frequency and amplitude to an electrochemical system; while the amplitude and the phase of the output current or voltage signal is monitored [1]. This technique allows obtaining a large number of properties of the studied electrochemical system such as the electrolyte ohmic resistance, the electrode properties (i.e. Charge transfer resistance and double layer capacitance) and the transport effects [2]. This is possible since EIS is able to deconvolve the different physic-chemical processes taking place in the system (i.e. charge transfer, mass transfer, etc...) [3]. This ability of EIS is the responsible that nowadays EIS has become a key experimental method in a large spectrum of fields [4]. This wide range of fields includes typically electrochemistry related fields as fuel cells [5-12], batteries [13-17], coatings [18-20], electrochemical sensors [21-26] and supercapacitors [27-31]. But it also includes fields not traditionally linked to electrochemistry as enzymatic kinetics [32], biochemistry [33-35], food quality control [36], cancer detection [37-38] and immunology [39-40], amongst others. This electrochemical technique was first introduced in wet-electrochemistry in the late sixties of last century; and then, during the seventies, it was adopted by the solid state researchers [41]. Today, EIS is widely used in both, liquid systems [42-43] and solid systems [44-45].

The name of "spectroscopy" is due to the fact that a range of perturbation frequencies are applied to the system, typically from the range of tens of $\mathrm{kHz}$ to the range of few $\mathrm{mHz}$ [46]. For each one of these applied frequencies, the impedance of the system is calculated. In this manner the EIS spectrum of the system is obtained: the impedance of the system as a function of the perturbation [2]. The impedance at a given angular frequency, $\omega$, is defined by Ohm's generalized law [1]:

$$
Z(\omega)=\frac{\mathcal{F}[U(t)]}{\mathcal{F}[I(t)]}
$$

Where $Z(\omega)$ stands for the complex impedance of the system at angular frequency $\omega=2 \pi f ; U(t)$ and $I(t)$ denote respectively the voltage signal and the current signal, in the time domain; and $\mathcal{F}$ corresponds with the Fourier transform operator. The impedance concept is the generalization of the DC electric resistance concept. Impedance is a complex magnitude: its module gives the electric resistance of the system (amplitude relation between the current and voltage signals), while its argument quantifies the time offset between both signals. 
Three conditions must be fulfilled for the complex Ohm's law, and thus the impedance concept, being valid: causality, linearity and stability [47]. If any of these four conditions is not met, then the obtained spectra may be biased, misleading and useless to extract proper conclusions on the studied system [48-49]. For this reason it is crucial to verify if an experimentally obtained EIS spectrum satisfies the four hypotheses, before performing the analysis itself [1].

A system is considered as a linear system when the relation between the perturbation signal and the output signal is given by a set of linear differential equations [50]. Consequently, in a linear system the superposition principle holds: the response to a linear combination of perturbations is a linear combination of the responses to each one of the individual perturbations [51]. Since electrochemical systems are in general governed by Buttler-Volmer's equation, they are generally highly nonlinear systems [52]. Therefore, in the case of electrochemical systems the linearity condition is achieved by applying a perturbation of low enough amplitude in order to guarantee that the nonlinear effects are negligible [53]. However very small perturbation amplitudes result in very low signal-to-noise ratios, that result in results with a high measurement error [1]. Consequently, the quality of the measurement of an EIS spectrum is determined by the trade-off between the linearity hypothesis fulfilment (low enough perturbation amplitude) and the maximization of the signal-to-noise ratio (high enough perturbation amplitude) [54].

There is a large number of works in literature that show that the non-fulfilment of the linearity condition leads to the generation of non-fundamental harmonics in the output signal. Some of such works are the works of Darowicki and co-workers [53, 5556], Victoria and Ramanathan [57], Diard and co-workers [58-63], and Van Gheem's team [64-65]. When a mono-frequency sinusoidal perturbation signal is applied to a linear system, a mono-frequency output signal of same frequency is obtained [66]. On the contrary, in the case of a nonlinear system, the output signal will be a superposition of different sine waves which frequencies are integer multiples of the fundamental frequency [67]. These non-fundamental harmonics introduce a systematic error in the measured EIS spectrum, which will result in a distortion of the spectrum; and consequently, in a bias in the results obtained from its analysis [68].

A lot of work has been done in literature in order to develop techniques to verify the fulfilment of the four fundamental hypotheses, in general [69-70]; and of the linearity condition, in particular [71]. Even a hybrid EIS measurement technique has been developed by Orazem's group: the perturbation amplitude is continually modified in order to achieve the linearity condition during the whole measurement [72]. 
Three main types of linearity assessment methods can be found in literature: the experimental methods (AC plots and Lissajous plots); the methods based in the harmonic analysis of the response signal; and the methods based in the KramersKronig relations [46]. On the one hand, the experimental methods allow assessing linearity in real time during the measurement. These methods include AC plots, resolution plots and Lissajous plots: all these plots allow identifying nonlinear behaviour by directly monitoring the raw signals in the time domain [1]. Nowadays, all EIS softwares include this type of tools, which can be displayed during data acquisition. On the other hand, methods based in harmonic analysis assess linearity by quantifying the level of non-fundamental harmonics in the output signal. Popkirov and Schindler presented one of these linearity assessment methods based on harmonic analysis [48, 67]. Another example of this type of linearity assessment methods can be found in the works of Pintelon's team [73-74]. Finally, the Kramers-Kronig relations are integral equations that relate the real and the imaginary part of complex quantities that meet the conditions of causality, finiteness, linearity and stability [2]. A great number of works show the power of Kramers-Kronig relations as EIS spectra validation tool. An extensive review of the main available methods to apply Kramers-Kronig transforms to validate EIS data was written by Agarwal and Orazem [69].

The main disadvantage of experimental and harmonic analysis based linearity assessment methods with respect to Kramers-Kronig based linearity assessment methods is that they cannot be applied to an already measured spectrum, if the raw signals have not been stored as well: while experimental methods are based on the monitoring of the raw signals themselves; harmonic analysis based methods require non-fundamental harmonic quantification, which is done by applying Fourier's transform to the raw signals. Meanwhile the Kramers-Kronig based methods only require the spectrum itself, and therefore they may be applied to an already measured spectrum even if the raw signals are not available.

In a previous work, an experimental spectrum quantitative validation technique based on Kramers-Kronig relations was presented [75]. In that work, the validation test was experimentally validated with respect to the stability condition: it was shown that the presented validation technique was not only able to detect the non-compliance of the stability condition; but it was also able to distinguish the inconsistent points from the consistent ones in a nonstationary situation. The aim of this work is to verify if the experimental spectrum quantitative validation technique is also able to detect the non-compliance of the linearity condition. In order to achieve this goal, the quantitative validation technique cited above was applied to the spectra of an alkaline hydrogen evolution electrochemical cell. These spectra were measured experimentally in the same operation conditions with different perturbation amplitudes; thereby obtaining a large range of degrees of nonlinearity in the system: ranging from the quasi 
linear behaviour (valid spectrum) to the highly nonlinear behaviour (non valid spectrum).

\section{Experimental spectrum quantitative validation technique}

\subsection{Overall methodology}

The experimental spectrum quantitative validation technique described in [75] consists in the combination of a Kramers-Kronig validation method by equivalent circuit fitting; with a Montecarlo error propagation method. Therefore, the experimental spectrum quantitative validation technique consists in two stages: firstly, the experimental spectrum is fit to an equivalent circuit that satisfies Kramers-Kronig relations. Then, in a second stage, the results of the fitting process (fitted circuit parameters and their corresponding standard deviations) are introduced in a Montecarlo based error propagation algorithm that builds a consistency zone for each one of the individual points of the experimental spectrum, for a given confidence level. The decision criterion is:

- The experimental points inside of the built consistency region can be considered as consistent with Kramers-Kronig relations, with the confidence level used to build the consistency regions. Therefore, these points can be considered as valid, and used in further analysis of the spectrum;

- On the contrary, the experimental points that lay outside the built consistency region can be considered as inconsistent with Kramers-Kronig relations, with the confidence level used to build the consistency regions. Consequently, it can be deduced that there are enough statistical evidences to conclude that these points are inconsistent points (do not satisfy at least one of the four hypotheses); and therefore, they should be deleted from any further analysis performed on the experimental spectrum.

A graphical summary of the overall methodology in which the experimental spectrum quantitative validation technique is based is shown in figure 2 of [75].

This quantitative validation technique is an hybrid method between Voigt's method [76-77] and the measurement model method developed by Orazem's group [69, 7886].

Voigt's method consists in validating the consistency of an experimental EIS spectrum by fitting it to an electric equivalent circuit that satisfies the Kramers-Kronig relations 
[69]: If the spectrum is well fitted by the equivalent circuit, then it is consistent with Kramers-Kronig relations and it can be considered as a valid spectrum. This method has two major drawbacks: it only allows to work with the spectrum as a whole (the whole spectrum is considered valid; or the whole spectrum is considered non valid); and the definition of the acceptance criterion of the test (the definition of "good" and "bad" fit) is non-trivial and depends on the studied system. The experimental spectrum quantitative validation technique overcomes both issues. The quantitative method sets the acceptance criterion for each point of the experimental spectrum through the Montecarlo algorithm. Moreover, since this method sets a different acceptance criterion for each individual point, it will detect inconsistent points individually rather than working with the spectrum as a whole.

In opposition, Orazem's measurement model method uses a generalized model that satisfies Kramers-Kronig relations (i.e. Voigt's model) as a filter for non-replicacy in impedance data [84]. This measurement model allows distinguishing between bias and stochastic errors, and obtaining the error structure of the experimental data [85]. The error structure is then used with a Montecarlo simulation to obtain an acceptance zone that allows discriminating between consistent and inconsistent points. The measurement model method is not only able to discriminate between consistent and inconsistent points individually; but it is also able to assess the noise level in the measurement. The major drawback of this method is that it requires replicating the impedance measurements; and therefore it cannot be applied to an already measured spectrum if it was not replicated during the original measurement. The experimental spectrum quantitative validation technique overcomes this issue since it does not require replicating the measurements.

\subsection{Circuit fitting}

As described in the previous subsection, the first step of the quantitative validation technique consists in fitting the experimental spectrum to an electric equivalent circuit that satisfies Kramers-Kronig relations. In this work, Voigt's circuit was used as the equivalent circuit to which the experimental spectra were fitted to. As it can be seen in figure 1, Voigt's circuit consists in a $R(R C)_{n}$ circuit: it is composed by $n$ parallel $R C$ subcircuits in series, with an individual resistance also in series. The main advantages of this circuit over other equivalent circuits are: its fulfilment of the Kramers-Kronig relations, and its versatility. As it was explained before, the fulfilment of the KramersKronig relations is a key feature that must have an equivalent circuit in order to be used in the quantitative validation technique. On the other hand, the Voigt's circuit is able to fit a wide range of spectra, just by modifying the number of $R C$ subcircuits, $n$. It can even fit spectra with inductive loops by considering $R C$ subcircuits with negative resistance and capacitance values [77]. It should be noted that the Voigt circuit does 
not have any physical meaning for the studied system; it is only part of a mathematical tool for spectrum validation. Consequently, negative values for resistances and capacitances can be accepted for the quantitative validation technique, even though they do not have any physical explanation.

Using the impedance expressions for resistors and capacitors, and the impedance combination rules [87], the following expressions were obtained for a $R(R C)_{n}$ circuit:

$$
\begin{gathered}
Z_{R(R C)_{n}}^{\prime}(\omega)=R_{1}+\sum_{i=1}^{n}\left(\frac{R_{R C_{i}}}{\left(R_{R C_{i}} \cdot C_{R C_{i}} \cdot \omega\right)^{2}+1}\right) \\
Z_{R(R C)_{n}}^{\prime \prime}(\omega)=-\sum_{i=1}^{n}\left(\frac{R_{R C_{i}}{ }^{2} \cdot C_{R C_{i}} \cdot \omega}{\left(R_{R C_{i}} \cdot C_{R C_{i}} \cdot \omega\right)^{2}+1}\right)
\end{gathered}
$$

Where $Z^{\prime}{ }_{R(R C)_{n}}$ and $Z^{\prime \prime}{ }_{R(R C)_{n}}$ denote respectively the real and the imaginary part of the impedance of the $R(R C)_{n}$ circuit; $R_{1}$ represents the individual resistance; and finally, $R_{R C_{i}}$ and $C_{R C_{i}}$ are the resistance and the capacitance of the $i$-th $R C$ subcircuit. Consequently, the vector of parameters associated with the $R(R C)_{n}$ circuit, $\vec{a}_{R(R C)_{n}}$, is a $2 n+1$ dimensional vector.

The fitting process consists in determining the vector $\vec{a}_{R(R C)_{n}}$ that minimizes the fitting error. In the EIS field, the parameter most commonly used to quantify the goodness of the fitting is the weighted sum of quadratic errors [1], $\chi^{2}$, which is defined as:

$$
\chi^{2}=\sum_{i=1}^{N_{p}}\left[\alpha_{i} \cdot\left(Z^{\prime} \exp \left(\omega_{i}\right)-Z_{R(R C)_{n}}^{\prime}\left(\omega_{i}\right)\right)^{2}+\beta_{i} \cdot\left(Z^{\prime \prime}{ }_{\exp }\left(\omega_{i}\right)-Z_{R(R C)_{n}}^{\prime \prime}\left(\omega_{i}\right)\right)^{2}\right]
$$

Where $N_{p}$ denotes the number of experimental points. Subscript $\exp$ refers to experimental points; whereas, subscript $R(R C)_{n}$ refers to model calculated points using equations (2) and (3). Finally, $\alpha_{i}$ and $\beta_{i}$ are the weight of the real and the imaginary part of the i-th point.

The quantitative validation technique is based in the hypothesis that the relative errors associated to measurement are constant in the whole frequency range [88]. The error structure that arises from this hypothesis is an error structure in which the absolute error for a given frequency is proportional to the impedance modulus at that frequency. The weighting strategy that emerges from this error structure is an inverseto-the-square weighting strategy: 


$$
\alpha_{i}=\beta_{i}=\frac{1}{\left|Z_{\exp }\left(\omega_{i}\right)\right|^{2}}
$$

This weighting strategy is very commonly used in EIS fittings [2]. The weighting strategy is a major difference between the quantitative validation technique and the measurement model method developed by Orazem and co-workers. In the measurement model method, rather than selecting a pre-defined error structure (as in the case of the validation method used in this work), the error structure is determined as an initial step of the method through measurement replication. Using the real error structure instead of a pre-defined one increases the power of the measurement model method, since it has been shown that the inverse-to-the-square weighting strategy is suboptimal for impedance data collected under galvanostatic modulation [82]. However a cost must be paid: measurement replication is required. The advantage of the quantitative validation technique is that it does not require measurement replication; and that therefore, it can be applied to existing spectra for which the measurements were not replicated.

In this work, the fitting process was done using a Levenberg-Marquardt minimization algorithm taking as the objective function the weighted sum of quadratic errors, given by equation (4). The fitting algorithm output consists of the best fit parameters (the vector $\vec{a}_{R(R C)_{n}}$ that minimizes $\chi^{2}$ ); and the uncertainty associated to each one of the obtained parameters, given by the standard error vector, $\vec{\sigma}_{R(R C)_{n}}$.

A key parameter of the fitting step of the quantitative validation technique is the selection of the number of subcircuits, $n$. As it was explained in [75], there is an optimal number of subcircuits. Below this optimal number, an increase in the number of subcircuits generates a significant increase in the goodness of the fitting, while not increasing significantly the uncertainty of the model parameters. On the contrary, above the optimum number, an increase in the number of subcircuits leads to a significant increase of the uncertainty of the model parameters, due to over fitting; and a negligible increase of the fitting goodness parameter. In this work, the optimum number of $R C$ subcircuits was determined individually for each spectrum, using the procedure described in the aforementioned reference.

\subsection{Montecarlo error propagation algorithm}

As described in the subsection 2.1, the second step of the quantitative validation technique consists in introducing the fitting results in a Montecarlo error propagation algorithm in order to propagate the fitting errors through the model. Firstly, a random vector of input model parameters is generated: 


$$
\vec{X}_{\text {ran }} \sim \mathcal{N}\left(\vec{a}_{R(R C)_{n}} ; \vec{\sigma}_{R(R C)_{n}}\right)
$$

Where $\mathcal{N}$ denotes the normal distribution. For the sake of simplicity a component-tocomponent vectorial notation has been used: the $i$-th component of vector $\vec{X}_{\text {ran }}$ is distributed according to a normal distribution of mean the $i$-th component of vector $\vec{a}_{R(R C)_{n}}$ and standard deviation the $i$-th component of vector $\vec{\sigma}_{R(R C)_{n}}$. Both, $\vec{a}_{R(R C)_{n}}$ and $\vec{\sigma}_{R(R C)_{n}}$ are known, since they were determined in the equivalent circuit fitting step. Therefore, each element of the equivalent electric circuit is assigned a random variable normally distributed around its fitted value, and with a standard deviation equal to its fitting uncertainty.

Secondly, the EIS spectrum of the equivalent circuit associated to the generated random vector is simulated: the generated random vector is introduced in equations (2) and (3) in order to calculate the real and the imaginary part of the impedance of the equivalent circuit associated to the generated random vector for every frequency in the experimental frequency range. This constitutes a single iteration of the Montecarlo algorithm.

A sufficiently large number of iterations have to be performed in order to ensure the convergence of the Montecarlo algorithm: for a large number of iterations the sample parameters tend to the population parameters [89]. Convergence graphs, as the ones shown in [75], were used in this work in order to monitor the convergence of the algorithm. Once convergence is reached, the consistency regions for each measured frequency can be built for the real part and the imaginary part, using the collection of simulated spectra:

$$
\begin{gathered}
Z^{\prime}\left(\omega_{i}\right) \in\left[\overline{Z^{\prime}}\left(\omega_{i}\right)-k \cdot s_{Z^{\prime}\left(\omega_{i}\right)} ; \overline{Z^{\prime}}\left(\omega_{i}\right)+k \cdot s_{Z^{\prime}\left(\omega_{i}\right)}\right] \\
Z^{\prime \prime}\left(\omega_{i}\right) \in\left[\overline{Z^{\prime \prime}}\left(\omega_{i}\right)-k \cdot s_{Z^{\prime \prime}\left(\omega_{i}\right)} ; \overline{Z^{\prime \prime}}\left(\omega_{i}\right)+k \cdot s_{Z^{\prime \prime}\left(\omega_{i}\right)}\right]
\end{gathered}
$$

While $\overline{Z^{\prime}}\left(\omega_{i}\right)$ and $\overline{Z^{\prime \prime}}\left(\omega_{i}\right)$ denote the sample means of the real and the imaginary part of the simulated impedance for angular frequency $\omega_{i}$ :

$$
\begin{aligned}
& \overline{Z^{\prime}}\left(\omega_{i}\right)=\frac{1}{n_{\text {ite }}} \cdot \sum_{k=1}^{n_{\text {ite }}} Z^{\prime}\left(\omega_{i}\right)_{k} \\
& \overline{Z^{\prime \prime}}\left(\omega_{i}\right)=\frac{1}{n_{\text {ite }}} \cdot \sum_{k=1}^{n_{\text {ite }}} Z^{\prime \prime}\left(\omega_{i}\right)_{k}
\end{aligned}
$$


Where $n_{\text {ite }}$ denotes the number of iterations.

$S_{Z^{\prime}\left(\omega_{i}\right)}$ and $s_{Z^{\prime \prime}\left(\omega_{i}\right)}$ stand for the sample standard deviations of the real part and the imaginary part of the impedance for angular frequency $\omega_{i}$ :

$$
\begin{aligned}
s_{Z^{\prime}\left(\omega_{i}\right)} & =\sqrt{\frac{1}{n_{i t e}-1} \cdot \sum_{k=1}^{n_{i t e}}\left(Z^{\prime}\left(\omega_{i}\right)_{k}-\overline{Z^{\prime}}\left(\omega_{i}\right)\right)^{2}} \\
s_{Z^{\prime \prime}\left(\omega_{i}\right)} & =\sqrt{\frac{1}{n_{\text {ite }}-1} \cdot \sum_{k=1}^{n_{i t e}}\left(Z^{\prime \prime}\left(\omega_{i}\right)_{k}-\overline{Z^{\prime \prime}}\left(\omega_{i}\right)\right)^{2}}
\end{aligned}
$$

Finally, $k$ represents the cover factor. This cover factor depends on the selected confidence level. Its value for different confidence levels can be obtained from any statistical book. A list of $k$ values for the typical confidence levels can be found in a previous work [75]. The cover factor determines $\mathbb{P}[$ Error $I]$, the probability of type I error. This probability corresponds with the probability of classifying as inconsistent a point that is consistent.

Once the confidence regions are built, the following criteria are applied: all the experimental points inside the consistency region can be considered to satisfy the Kramers-Kronig relations (with a given confidence level), and therefore are consistent and can be used in the spectrum further analysis. In contrast, any point outside the consistency region does not satisfy the Kramers-Kronig relations (with a given confidence level), and consequently does not satisfy at least one of the four conditions, hence it has to be from further analysis of the spectrum.

\subsection{Practical implementation}

The quantitative validation technique can be easily automated in any programing language. In this work, it was fully implemented in Labview ${ }^{\circledR}$ : the developed Labview $\AA$ program takes as input the output of NOVA®, which was the software used to control the EIS measurement system. Then, the program fits the experimental spectrum to Voigt circuits with different number of subcircuits. From the results of these fittings, the program determines the optimum number of subcircuits. Finally, the program runs the Montecarlo algorithm with the results of the fitting with the optimum number of subcircuits, in order to build the consistency zones.

The computational time required to run the quantitative validation technique on an experimental EIS spectrum is of about $30 \mathrm{~min}$ in a typical laptop [75]. Therefore, this 
validation algorithm can be easily implemented into routine analysis of the experimental results, without considerably overextending the required time for the preliminary analysis of the experimental results; and without the need of replicating the measurements.

\section{Experimental work}

A highly nonlinear electrochemical system was needed for this work in order to be able to obtain a large range of degrees of nonlinearity by changing the perturbation amplitude. The selected system was the cathodic electrode of an alkaline water electrolyser. This system was described in detail by Herraiz-Cardona and co-workers [90-93]. The experimental work of this work consisted in the measurement of the EIS spectra of the cathodic electrode of an alkaline water electrolyser using different perturbation amplitudes, and thus obtaining different nonlinearity degrees.

Figure 2 shows the experimental setup that was used. It consists in a 3 electrode electrochemical cell patented by the Dpto. Ingeniería Química y Nuclear of the Universitat Politècnica de València [94].

On the one hand, the working electrode was one of the electrodes developed by Herraiz-Cardona and co-workers. It consisted in a nickel electrode produced at very high current densities using the protocol described in [91]. This procedure yields rough porous electrodes with very high active areas. On the other hand, the counterelectrode was a nickel foam with very high surface area (Incofoam $\AA 0.17 \mathrm{~cm}$ thick and 50 pores per linear inch). Finally, a commercial $\mathrm{Ag} / \mathrm{AgCl}(3 \mathrm{M} \mathrm{KCl}$ ) electrode was used as the reference electrode. The electrochemical cell was filled with a $30 \mathrm{wt} . \% \mathrm{KOH}$ oxygen free solution, that played the electrolyte role.

The EIS measurements were performed using an Autolab® $302 \mathrm{~N}$ potentiostat/galvanostat equipped with a FRA module. The measurement equipment was controlled using NOVA $\AA$ software. The measurements were taken in the frequency range of $10 \mathrm{kHz}-5 \mathrm{mHz}$, with 10 frequencies per decade. The measurement parameters used in this work are given in table 1 . These parameters were optimized using the methodology presented in a previous work [95], where the meaning of each measurement parameter was widely explained.

All the experiments were performed in galvanostatic mode and in the same operation conditions: at $30^{\circ} \mathrm{C}$ and a DC current of $-10 \mathrm{~mA}$. This operation DC current was selected, since in previous works, it was observed that it corresponded to the most nonlinear operation point of the system at $30^{\circ} \mathrm{C}$. In each experiment, perturbations of 
different amplitudes were used. The perturbation amplitude range that was considered in this work was from $2 \mathrm{~mA}$ to $10 \mathrm{~mA}$, with a step size of $1 \mathrm{~mA}$. The lowest perturbation amplitude ( $2 \mathrm{~mA}$ ) was selected since it is known from preliminary works that this perturbation amplitude is the optimum perturbation amplitude of the system: the highest perturbation amplitude for which the linearity condition is fulfilled. The highest perturbation amplitude $(10 \mathrm{~mA})$ was selected since it is the maximum amplitude that can be used for a DC current of $-10 \mathrm{~mA}$ that guarantees that the working electrode works as cathode during the whole measurement cycle.

In order to ensure the reproducibility of the results it is critical to ensure that the surface of the electrode is in similar conditions in all the experiments. To achieve this, a pre-treatment was applied to the working electrode before each experiment. This pre-treatment consists in the application of a $-1.6 \mathrm{~V}$ ( $\mathrm{vs} . \mathrm{Ag} / \mathrm{AgCl}$ ) potential during 30 minutes. The purpose of this pre-treatment is to reduce any oxide film that could exist on the surface of the porous electrode [92]; thereby guaranteeing that the surface of the working electrode was in similar conditions in all the experiments.

Measurements were obtained in triplicate in order to control the reproducibility of the obtained results. Replicates of each measurement were not performed sequentially; instead, the experiments were done in three different blocks as shown in table 2, which gives the temporary order in which the experiments were performed. As it can be seen in the table mentioned above, the order of the experiments inside each block was randomized; instead of performing the experiments in order of increasing or decreasing amplitude. The random order strategy was selected since randomization makes the factors of time and amplitude orthogonal: this means that it allows to distinguish the effects of the perturbation amplitude from the effects of possible time drifts. On the contrary, if an increasing amplitude strategy is used, it is not possible to know if the observed trends are due to a time drift of the system or to the effect of the perturbation amplitude. 


\section{$\underline{\text { 4. Results and discussion }}$}

\subsection{Nyquist diagrams}

Figure 3 shows the experimental EIS spectra obtained with each one of the perturbation amplitudes. It should be noted that all the spectra were measured in the same operation conditions; and the only thing that changed from one to another was the applied perturbation amplitude. All the spectra present a depressed capacitive semicircle. Actually, this semicircle is formed by two overlapping semi-circles, a high frequency one and an intermediate frequency one [92]. The high frequency semi-circle is related to the porosity of the electrode surface; whereas the intermediate frequency semi-circle is related to the Hydrogen Evolution Reaction (HER) kinetics [92]. In addition, all the spectra except the $10 \mathrm{~mA}$ one, present the beginning of a low frequency inductive semi-circle. This low frequency inductive semi-circle is due to the adsorption/desorption processes of intermediate species on the electrodes. Furthermore, a displacement of the spectra from the origin of Nyquist plot can be observed. This is related to the resistance of the electrolyte between the working and the reference electrodes. Finally, small inductive effects can be observed at high frequencies. These high frequency effects are measurement artefacts due to the cable connections [96-97]. Since they are due to the connections themselves and not to the studied system, these high frequency inductive effects were not taken into account in this work.

The results obtained in the three replicates of each experiment are nearly identical: the results have a very high reproducibility. For clarity purposes, since they are nearly identical, only the results of one of the replicate blocks are presented in figure 2 . Since a random order strategy was used, the fact that the results are highly reproducible implies that no significant time drift took place during the experiments. Consequently, the observed variation of the experimental spectra is only due to nonlinear effects and not to a time drift or a non-stationarity of the system.

Although the general shape of the spectrum is the same for the different perturbation amplitudes (except for a perturbation amplitude of $10 \mathrm{~mA}$, for which even the general shape varies), a significant variation of the spectra with the perturbation amplitude can be observed. A systematic distortion of the system spectrum is observed when the perturbation amplitude is increased: an amplitude increase leads to a significant and systematic expansion of the intermediate frequency capacitive semi-circle. In the extreme case of a $10 \mathrm{~mA}$ perturbation amplitude, the distortion is so important that it causes a change in the overall shape of the spectrum: the low frequency inductive semi-circle is no longer observed. This systematic distortion of the EIS spectrum with an increase of the perturbation amplitude is due to 
the generation of nonlinear effects by the studied system, which in this case is a highly nonlinear system. The expansion of the low frequency zone of the EIS spectrum of an electrochemical electrode with an increase of the perturbation amplitude was already observed by Diard and co-workers [61-62], and is consistent with their theoretical formulation [59]. Moreover, in a previous work it was shown that the observed systematic distortion of the EIS spectrum with an increase of the perturbation amplitude was due to the emergence of nonlinear effects for amplitudes larger than a critical perturbation amplitude, which is of $2 \mathrm{~mA}$ for the system studied in this work [98].

The fact that the spectrum distortion is only observed in the intermediate and low frequency zones is due to the fact that nonlinear effects only appear under a threshold frequency. For frequencies above the threshold frequency the nonlinear effects are negligible even for very large perturbation amplitudes. Thus, the part of the EIS spectrum related with frequencies higher than the threshold frequency will not be distorted by an amplitude increase, even for large amplitudes. On the contrary, for frequencies below the threshold frequency the nonlinear effects are not negligible for amplitudes above a critical perturbation amplitude. Consequently, a significant distortion of the EIS spectrum will be observed in the frequency zone corresponding with frequencies below the threshold frequency, for perturbation amplitudes above the critical amplitude. The existence of a frequency threshold above which the nonlinear effects are negligible was already observed by Hirschorn and co-workers [54, 99].

The threshold frequency of the cathodic electrode of an alkaline water electrolyser system, studied in this work, can be estimated from figure 3. It corresponds with the frequency above which the effect of the perturbation amplitude on the spectrum is significant: this can be approximated to the frequency above which the spectra diverge in figure 3. Consequently, the threshold frequency of the studied system is in the range of $5 \mathrm{~Hz}-7 \mathrm{~Hz}$. This threshold frequency was determined in a previous work using a total harmonic distortion based linearity assessment method [98]. The threshold frequency obtained with the linearity assessment method was consistent with the threshold frequency estimated from figure 3.

Finally, it can be observed that the $50 \mathrm{~Hz}$ point presents a high variability from one spectrum to another. However, there is no systematic pattern in its variation. The variation of the $50 \mathrm{~Hz}$ point is not due to the effect of the perturbation amplitude; instead, it is due to noise. This frequency presents higher noise than the rest of the spectrum due to the electric coupling of the measurement instrument with the electric supply grid. This coupling is responsible for the random variability of the $50 \mathrm{~Hz}$ point. 


\subsection{Consistency zones}

The quantitative validation technique presented in section 2 was applied to each one of the experimental spectra individually. The number of iterations used for the Montecarlo algorithm was 100000 in every case, since this value guaranteed the convergence of the method, as it was observed in [75]. The obtained results are shown in figures 4 to 9 . These figures give the real and imaginary plots for each case. The experimental points are represented with the built consistency regions overlaid. The represented consistency zones correspond to a confidence level of $95.45 \%$. They are defined by an upper limit and lower one. These representations allow to easily detect the inconsistent points, just by identifying the experimental points outside of the corresponding consistency region. Since the results obtained for perturbation amplitudes from $2 \mathrm{~mA}$ to $6 \mathrm{~mA}$ are very similar, only the results of $2 \mathrm{~mA}$ and $3 \mathrm{~mA}$ are presented for the sake of simplicity.

It can be observed that the quantitative validation technique does not detect any inconsistent point for perturbation amplitudes from $2 \mathrm{~mA}$ to $7 \mathrm{~mA}$. However, it can be observed that the consistency regions narrow with increasing perturbation amplitude. On the contrary, the quantitative validation technique detects inconsistent points for perturbation amplitudes of $8 \mathrm{~mA}, 9 \mathrm{~mA}$ and $10 \mathrm{~mA}$. In the three cases, the identified inconsistent points are in the intermediate/low frequency region. As it was discussed in the previous subsection, the nonlinear distortion of EIS spectra only occurs in the frequency range below the transition frequency. Therefore, the series of inconsistent points detected by the quantitative validation technique correspond with part of the distortion due to nonlinear effects. Consequently, the quantitative validation technique is able to detect nonlinearities, but only in the case of very significant nonlinear effects ( $8 \mathrm{~mA}$ to $10 \mathrm{~mA}$ ). It does not detect slight nonlinearities ( $3 \mathrm{~mA}$ to 7 $m A)$. Urquidi-Macdonald and co-workers already observed that the Kramers-Kronig relations were highly insensitive to nonlinearity [50]. More recently, it has been shown that Kramers-Kronig relations are only sensitive to nonlinearities if at least part of the spectrum has been measured in a range above the threshold frequency of the system; and even then, the sensitivity is not very high [99]. In this case, the threshold frequency of the system ( 5 to $7 \mathrm{~Hz}$ ) is encompassed by the measurement frequency range $(10 \mathrm{kHz}$ to $5 \mathrm{mHz}$ ); therefore the requirement proposed by Hirschorn and Orazem is fulfilled in this work. Consequently, under these conditions, the KramersKronig relations are sensitive to nonlinearities, though there sensitivity is not very high. Since the quantitative validation technique is based on Kramers-Kronig relations, it is normal that it is only able to detect big nonlinearities and not slight ones; and only if the threshold frequency is encompassed by the measurement frequency range. This is consistent with the observations extracted from figures 4 to 9 . 
In the $8 \mathrm{~mA}$ case, the quantitative validation technique only detects the inconsistent points in the imaginary plot; and not in the real plot. In the $9 \mathrm{~mA}$ and the $10 \mathrm{~mA}$ cases, it detects inconsistent points in both the real and the imaginary plots. However, in both cases the detected inconsistent point with the highest frequency is identified in the imaginary plot and not in the real plot. This suggests that the imaginary component is more sensitive to nonlinearities (at least for this system); and therefore, the imaginary consistency region has a higher detection power.

In the $8 \mathrm{~mA}, 9 \mathrm{~mA}$ and $10 \mathrm{~mA}$ cases, the frequency of the inconsistent point with the highest frequency is around $1 \mathrm{~Hz}$, as it can be seen in figures 7.b, 8.b and 9.b. This value corresponds with the estimation of the threshold frequency determined using the quantitative validation technique, since it is the highest frequency for which the measured impedance fails to satisfy the linearity condition according to the quantitative validation technique; and by definition, the threshold frequency is the frequency above which the system does not present nonlinear behaviour even for very large perturbation amplitudes. In subsection 4.1, the threshold frequency of the system was estimated to be around $5-7 \mathrm{~Hz}$. It can be observed that the order of magnitude of the threshold frequency determined using the quantitative validation technique is of the same order of magnitude than the threshold frequency estimated from the EIS spectra themselves. It can be deduced that the quantitative validation technique allows to properly estimate the threshold frequency of a given system with the proviso that the nonlinearity is large enough, since the method is not able to detect slight nonlinearities.

The whole study was repeated using potentiostatic modulation in order to determine if the type of modulation affected the sensitivity of the quantitative validation technique to nonlinearities. Exactly the same trends observed in galvanostatic mode, and presented in this work, were observed in potentiostatic mode. It can be deduced that the type of modulation does not significantly affect the sensitivity of the quantitative validation technique to nonlinearities. 


\section{$\underline{\text { 5. Conclusions }}$}

In conclusion, the quantitative validation technique manages to validate the experimental impedance spectra by building a consistency region that allows distinguishing the consistent points of an experimental spectrum from the inconsistent ones. In this work, it has been proved experimentally that the quantitative validation technique is able to detect severe nonlinearities; but it is not sensible enough to detect slight nonlinearities. The low sensitivity to nonlinearity of the quantitative validation technique is inherited from the Kramers-Kronig relations, which have been proved not to be very sensitive to the failure to fulfil the linearity condition. In addition, in the case of severe nonlinearities, the quantitative validation technique gives a good estimation of the transition frequency of the system, above which the system does not present a nonlinear behaviour even for large perturbation amplitudes.

First, the advantage of the proposed method over the traditional equivalent circuit fitting KK test (Voigt's method) is that the proposed method is a quantitative method, whereas Voigt's test is a rather subjective method: what is the limit between a good fit and a bad fit? The proposed method gives an objective quantitative criterion for acceptance/rejection of points; and it allows to discriminate individual points, rather than working with the spectrum as a whole. Second, the advantage of the proposed method over the existing linearity assessment tools available in literature, such as Lissajous plots and FFT based linearity assessment methods, is that it can be performed in already measured spectra for which only the spectrum is available (nor the measured raw signals in the time domain, nor the signals in the frequency domain are available). Furthermore, the proposed method does not have big computational requirements. These characteristics make the quantitative validation technique a good candidate to be automated and included in routine preliminary analysis of EIS spectra: before the analysis of any EIS spectra, the proposed method can be applied to it in order to detect the presence of high nonlinearities during the measurement that may have distorted the spectrum, and thus, bias the obtained results. The consistency regions will discriminate the consistent points from the inconsistent ones, allowing to remove the inconsistent ones from further analysis of the EIS spectrum. 


\section{Nomenclature}

\section{$\underline{\text { Latin letters }}$}

$\begin{array}{ll}C & \text { Capacitance }(F) \\ n & \text { Number of RC subcircuits in Voigt circuit } \\ n_{i t e} & \text { Number of iterations } \\ N_{p} & \text { Number of points in the impedance spectrum } \\ R & \text { Resistance }(\Omega) \\ \mathbb{P} & \text { Probability operator } \\ S & \text { Sample standard deviation } \\ \vec{X}_{\text {ran }} & \text { Random vector of input parameters } \\ \bar{X} & \text { Sample mean } \\ Z & \text { Complex impedance }(\Omega) \\ Z^{\prime} & \text { Impedance real part }(\Omega) \\ Z^{\prime \prime} & \text { Impedance imaginary part }(\Omega)\end{array}$

\section{Greek letters}

$\alpha \quad$ Real part weight factor

$\beta \quad$ Imaginary part weight factor

$\chi^{2} \quad$ Sum of weighed squared residuals of the model $\left(\Omega^{2}\right)$

$\omega \quad$ Angular frequency $\left(\mathrm{rad} \cdot \mathrm{s}^{-1}\right)$

\section{$\underline{\text { Subscripts }}$}

exp Experimental spectrum points

$R C_{i} \quad$ i-th $\mathrm{RC}$ subcircuit of Voigt circuit

$R(R C)_{n} \quad$ Voigt circuit with $n$ RC subcircuits 


\section{Acknowledgments}

The authors are very grateful to the Generalitat Valenciana for its economic support in form of Vali+d grant (Ref: ACIF-2013-268). 


\section{$\underline{\text { 8. References }}$}

[1] M.E. Orazem, B. Tribollet, Electrochemical impedance spectroscopy, John Wiley \& Sons, New Jersey, 2008.

[2] E. Barsoukov, J.R. Macdonald, Impedance spectroscopy. Theory, experiment and applications. John Wiley \& Sons, New Jersey, 2005.

[3] F. Ciucci, C. Chen, Analysis of electrochemical impedance spectroscopy data using the distributionof relaxation times: A bayesian and hierchical Bayesian approach, Electrochim. Acta 167 (2015) 439.

[4] D.D. Macdonald, Reflections on the history of electrochemical impedance spectroscopy, Electrochim. Acta 51 (2006) 1376.

[5] C. Boyer, S. Gamburzev, O. Velev, S. Srinivasan, A.J. Appleby, Measurement of proton conductivity in the active layer of PEM fuel cell gas diffusion electrodes, Electrochim. Acta 43 (1998) 3703.

[6] M. Lang, C. Auer, A. Eismann, P. Szabo, N. Wagner, Investigation of solid oxide fuel cell short stacks for mobile applications by electrochemical impedance spectroscopy, Electrochim. Acta 53 (2008) 7509.

[7] M. Mamlouk, K. Scott, Analysis of high temperature polymer electrolyte membrane fuel cell electrodes using electrochemical impedance spectroscopy, Electrochim. Acta 56 (2011) 5493.

[8] D. Vladikova et al., Impedance spectroscopy studies of dual membrane fuel cell, Electrochim. Acta 56 (2011) 7955.

[9] O. Reid, F.S. Easton, E.B. Saleh, Determining electrochemically active surface area in PEM fuel cell electrodes with electrochemical impedance spectroscopy and its applicatiom to catalyst durability, Electrochim. Acta 114 (2013) 278.

[10] Y. Fu et al., Heterogeneous electrocatalysis in porous cathodes of solid oxide fuel cells, Electrochim. Acta 159 (2015) 71.

[11] A. Baricci A. Casalegno, A simple analytical approach to simulate the electrochemical impedance response of flooded agglomerates in polymer fuel cells, Electrochim. Acta 157 (2015) 324. 
[12] D. Kashyap et al., Fabrication of vertically aligned copper nanotubes as a novel electrode for enzymatic biofuel cells, Electrochim. Acta 167 (2015) 213.

[13] S.S. Zhang, K. Xu, T.R. Jow, EIS study on the formation of solid electrolyte interface in Li-ion battery, Electrochim. Acta 51 (2006) 1636.

[14] J. Yun et al., In-situ electrochemical coating of Ag nanoparticles onto praphite electrode with enhanced performance for Li-ion batteries, Electrochim. Acta 155 (2015) 396.

[15] T. Yokoshima et al., Application of electrochemical impedance spectroscopy to ferri/ferrocyanide redox couple and lithium ion battery systems using a square wave as signal input, Electrochim. Acta 180 (2015) 922.

[16] J. Huang, H. Ge, Z. Li, J. Zhang, Dynamic electrochemical impedance spectroscopy of a three-electrode lithium-ion battery during pulse charge and discharge, Electrochim. Acta 176 (2015) 311.

[17] E. Samadani et al., Empirical modeling of lithium-ion batteries based on electrochemical impedance spectroscopy tests, Electrochim. Acta 160 (2015) 169.

[18] A.C. Ciubotariu, L. Benea, M. Dragan, V. Lakatos-Varsanyl, Electrochemical impedance spectroscopy and corrosion behaviour of Al203-Ni nano composite coatings, Electrochim. Acta 53 (2008) 4557.

[19] J. Molina et al., Electrochemical characterization of electrochemically reduced graphene coatings on platinum. Electrochemical study of dye adsorption, Electrochim. Acta 166 (2015) 54.

[20] A.S. Nguyen et al., Impedance analysis of the distributed resistivity of coatings in dry and wet conditions, Electrochim. Acta 179 (2015) 452.

[21] B. Pejcic, R. De Marco, Impedance spectroscopy: Over 35 years of electrochemical sensor optimization, Electrochim. Acta 51 (2006) 6217.

[22] R. Pauliukaite, M.E. Ghica, O. Fatibello-Filho, C.M.A. Brett, Electrochemical impedance studies of chitosan-modified electrodes for application in electrochemical sensors and biosensors, Electrochim. Acta 55 (2010) 6239.

[23] L. Manjakkal et al., Electrochemical impedance spectroscopic analysis of RuO2 based thick film pH sensors, Electrochim. Acta 168 (2015) 246. 
[24] F. Long, Z. Zhang, J. Wang, L. Yan, B. Zhou, Cobalt-nickel bimetalic nanparticles decorated graohene sensitized imprinted electrochemical sensor for determination of octylphenol, Electrochim. Acta 168 (2015) 337.

[25] T. Yoetz-Kopelman, Y. Ram, A. Freeman, Y. Shacham-Diamand, Faradic impedance spectroscopy for detection of small molecules binding using the avidin-biotin model, Electrochim. Acta 173 (2015) 630.

[26] G. Bhanjana, N. Dilbaghi, R. Kumar, A. Umar, S. Kumar, Sno2 quantum dots as novel platform for electrochemical sensing of cadmium, Electrochim. Acta 169 (2015) 97.

[27] E. Karaca, N. Ozcicek, K. Pekmez, Galvanostatic deposition of polypyrrole in the presence of tartaric acid for electrochemical supercapacitor, Electrochim. Acta 147 (2014) 545.

[28] Y. Zhang, Y. Mo, Preparation of MnO2 electrodes coated by Sb-doped SnO2 and their effect on electrochemical performance of supercapacitors, Electrochim. Acta 142 (2014) 76.

[29] J. Chen, Z. Xia, H. Li, Q. Li, Y. Zhang, Preparation of highly capacitive polyaniline/black $\mathrm{TiO} 2$ nanotubes as supercapacitor electrode by hidrogenation and electrochemical deposition, Electrochim. Acta 166 (2015) 174.

[30] M.F. Dupont, S.W. Donne, A step potential electrochemical spectroscopy analysis of electrochemical capacitor electrode performance, Electrochim. Acta 167 (2015) 268.

[31] G. Jin et al., Strongly coupled graphene/Mn3O4 composite with enhanced electrochemical performance for supercapacitor electrode, Electrochim. Acta 178 (2015) 689.

[32] T. Vidakovic-Koch, V.K. Mittal, T.Q.N. Do, M. Varnicic, K. Sundmacher, Application of electrochemical impedance spectroscopy for studying of enzyme kinetics, Electrochim. Acta 110 (2013) 94.

[33] M. Naumowicz, A.D. Petelska, Z.A. Figaszewski, Impedance spectroscopic investigation between phosphatidyethanolamine and alfa-tocopherol in bilayer membranes, Electrochim. Acta 54 (2009) 1089. 
[34] K. Mallaiya, S. Rameshkumar, S.S Subramanian, S. Ramalingam, T. Ramachandran, Electrochemical impedance studies on the interaction of midazolam with planar lipid bilayer, Electrochim. Acta 138 (2014) 360.

[35] L.M. Bravo-Anaya et al., The scaling of electrochemical parameters of DNA aqueous solutions with concentration and temperature through an electrochemical impedance spectroscopy study, Electrochim. Acta 167 (2015) 311.

[36] J.T. Liu, K. Settu, J.Z. Tsai, C.J. Chen, Impedance sensor for rapid enumeration of E. Coli in milk samples, Electrochim. Acta 182 (2015) 89.

[37] R. Wang, J. Di, J. Ma, Z. Ma, Highly sensitive detection of cancer cells by electrochemical impedance spectroscopy, Electrochim. Acta 61 (2012) 179.

[38] H. Quan et al., Electrochemical detection of carcinoembryonic antigen based on silver nanocluster/horseradish peroxidase nanocomposite as signal probe, Electrochim. Acta 176 (2015) 893.

[39] A. Sargent, O.A. Sadik, Monitoring antibody-antigen reactions at conducting polymer-based immunosensors using impedance spectroscopy, Electrochim. Acta 44 (1999) 4667.

[40] W.M. Hassen, V. Duplan, E. Frost, J.J. Dubowski, Quantification of influenza A virus in the presence of extraneous protein using electrochemical impedance spectroscopy, Electrochim. Acta 56 (2011) 8325.

[41] B.A. Boukamp, Fourier transform distribution function of relaxation times: application and limitations, Electrochim. Acta 154 (2015) 35.

[42] V. Shkirskiy, K. Ogle, A novel coupling of electrochemical impedance spectroscopy with atomic emission spectroelectrochemistry: Application to the open circuit dissolution of zinc, Electrochim. Acta 168 (2015) 167.

[43] E.A. Franceschini, G.I. Lacconi, H.R. Corti, Kinetics of the hydrogen evolution on nickel in alkaline solution: new insight from rotating disk electrode and impedance spectroscopy analysis, Electrochim. Acta 159 (2015) 210.

[44] C.C.H Tran, C. Autret, C. Damas, B. Claude-Montigny, J. Santos-Peña, An electrochemical study of Fe1.18Sb1.82 as negative electrode for sodium ion batteries, Electrochim. Acta 182 (2015) 11. 
[45] S.Y Lu et al., The effect of tempering temperature on the microstructure and electrochemical properties of a 13 wt.\% Cr-type martensitic stainless steel, Electrochim. Acta 165 (2015) 45.

[46] A. Lasia, Electrochemical impedance spectroscopy and its applications, Springer London, 2014.

[47] D.D. Macdonald, E. Sikora, Characterizing electrochemical systems in the frequency domain, Electrochim. Acta 43 (1997) 87, 1997.

[48] G.S. Popkirov, R.N. Schindler, Optimization of the perturbation signal for electrochemical impedance spectroscopy in the time domain, Rev. Sci. Instrum. 64 (1993) 3111.

[49] J.E. Garland, C.M. Pettit, D. Roy, Analysis of experimental constraints and variables for time resolved detection of Fourier transform electrochemical impedance spectra, Electrochim. Acta 49 (2004) 2623.

[50] M. Urquidi-Macdonald, S. Real, D.D. Macdonald, Applications of Kramers-Kronig transforms in the analysis of electrochemical impedance data - III. Stability and linearity, Electrochim. Acta 35 (1990) 1559.

[51] W. Aperador, J.H. Bautista Ruiz, O. Pardo Cuervo, Electrochemical behaviour of thin films of $\mathrm{CrN} / \mathrm{Cr}$ obtained varying the bias potential, Rev. Mex. Ing. Quim. 11 (2012) 145.

[52] K. Darowicki, Linearization in impedance measurements, Electrochim. Acta 42 (1997) 1781.

[53] K. Darowicki, The amplitude analysis of impedance spectra, Electrochim. Acta 40 (1995) 439.

[54] B. Hirschorn, B. Tribollet, M.E. Orazem, On selection of the perturbation amplitude required to avoid nonlinear effects in impedance measurements, Israel J. Chem. 48 (2008) 133.

[55] K. Darowicki, Frequency dispersion of harmonic components of the current of an electrode process, J. Electroanal. Chem. 394 (1995) 81.

[56] J. Smulko, K. Darowicki, Nonlinearity of electrochemical noise caused by pitting corrosion, J. Electroanal. Chem. 545, p. 59, 2003. 
[57] S.N. Victoria, S. Ramanathan, Effect of potential drift and ac amplitude on the electrochemical impedance spectra, Electrochim. Acta 56 (2011) 2606.

[58] J.P Diard, B. Le Gorrec, C. Montella, Theoretical formulation of the odd harmonic test criterion for EIS measurements, J. Electroanal. Chem. 377 (1994) 61.

[59] J.P. Diard, B. Le Gorrec, C. Montella, Impedance measurement errors due to nonlinearities - I. Low frequency impedance measurements, Electrochim. Acta 39 (1994) 539.

[60] J.P. Diard, B. Le Gorrec, C. Montella, Deviation from the polarization resistance due to non-linearity. I-Theoretical formulation, J. Electroanal. Chem. 432 (1997) 27.

[61] J.P. Diard, B. Le Gorrec, C. Montella, Deviation of the polarization resistance due to non-linearity - II. Application to electrochemical reactions, J. Electroanal. Chem. 432 (1997) 41.

[62] J.P. Diard, B. Le Gorrec, C. Montella, Deviation of the polarization resistance due to non-linearity - III. Polarization resistance determination from non-linear impedance measurements, J. Electroanal. Chem. 432 (1997) 53.

[63] J.P. Diard, B. Le Gorrec, C. Montella, Non-linear impedance for a two-step electrode reaction with an intermediate adsorbed species, Electrochim. Acta 42 (1997) 1503.

[64] E. Van Gheem et al., Electrochemical impedance spectroscopy in the presence of non-linear distortions and non-stationary behaviour. Part I: Theory and validation, Electrochim. Acta 49 (2004) 4753.

[65] E. Van Gheem et al., Electrochemical impedance spectroscopy in the presence of non-linear distortions and non-stationary behaviour. Part II. Application to crystallographic pitting corrosion of aluminium, Electrochim. Acta 51 (2006) 1443.

[66] M. Kiel, O. Bohlen, D.U. Sauer, Harmonic analysis for identification of nonlinearities in impedance spectroscopy, Electrochim. Acta 53 (2008) 7367.

[67] G.S Popkirov, R.N. Schindler, Effect of sample nonlinearity on the performance of time domain electrochemical impedance spectroscopy, Electrochim. Acta 40 (1995) 2511. 
[68] W. Lai, Fourier analysis of complex impedance (amplitude and phase) in nonlinear systems: A case study of diodes, Electrochim. Acta 55 (2010) 5511.

[69] P. Agarwal, M.E. Orazem, L.H. Garcia-Rubio, Application of measurement models to impedance spectroscopy - III. Evaluation of consistency with the Kramers-Kronig relations, J. Electrochem. Soc. 142 (1995) 4159.

[70] J.M. Esteban, M.E. Orazem, On the application of the Kramers-Kronig relations to evaluate the consistency of electrochemical impedance data, J. Electrochem. Soc. 138 (1991) 67.

[71] F. Fasmin, R. Srinivasan, Detection of nonlinearities in electrochemical impedance spectra by Kramers-Kronig transforms, J. Solid State Electr. 19 (2015) 1833.

[72] P. Wojcik, P. Agarwal, M.E. Orazem, A method for maintaining a constant potential variation during galvanostatic regulation of electrochemical impedance measurements, Electrochim. Acta 41 (1997) 977.

[73] R. Pintelon, E. Louarroudi, J. Lataire, Detecting and quantifying the nonlinear and time-variant effects in FRF measurements using periodic excitations, IEEE T. Instrum. Meas. 62 (2013) 3361.

[74] R. Pintelon, E. Louarroudi, J. Lataire, Nonparametric time-variant frequency response function estimates using arbitrary excitations, Automatica 51 (2015) 308.

[75] J.J. Giner-Sanz, E.M. Ortega, V. Perez-Herranz, Montecarlo based quantitative Kramers-Kronig test for PEMFC impedance spectrum validation, Int. J. Hydrogen Energ. 40 (2015) 11279.

[76] B.A. Boukamp, J.R. Macdonald, Alternatives to Kronig-Kramers transformation and testing, and estimation of distributions, Solid State lonics 174 (1994) 85.

[77] B.A. Boukamp, A linear Kronig-Kramers transform test for immittance data validation, J. Electrochem. Soc. 142 (1995) 1885.

[78] P. Agarwal, M.E. Orazem, L.H. Garcia-Rubio, Measurement models for electrochemical impedance spectroscopy: I. Demonstration of applicability, J. Electrochem. Soc. 139 (1992) 1917. 
[79] P. Agarwal, O.D. Crisalle, M.E. Orazem, L.H. Garcia-Rubio, Application of measurement models to impedance spectroscopy: II. Determination of the stochastic contribution to the error structure, J. Electrochem. Soc. 142 (1995) 4149.

[80] P. Agarwal, M.E. Orazem, L.H. Garcia-Rubio, The influence of error structure on interpretation of impedance spectra, Electrochim. Acta 41 (1996) 1017.

[81] M.E. Orazem, J.M. Esteban, O.C. Moghissi, Practical applications of the KramersKronig relations, Corrosion 47 (1991) 248.

[82] M.E. Orazem, P. Agarwal, C. Deslouis, B. Tribollet, Application of measurement models to electrohydrodynamic impedance spectroscopy, J. Electrochem. Soc. 143 (1996) 948.

[83] M.E. Orazem, P. Shukla, M.A. Membrino, Extension of the measurement model approach for deconvolution of underlying distributions for impedance measurements, Electrochim. Acta 47 (2002) 2027.

[84] M.E. Orazem, A systematic approach toward error structure identification for impedance spectroscopy, J. Electroanal. Chem. 572 (2004) 317.

[85] M.E. Orazem, B. Tribollet, An integrated approach to electrochemical impedance spectroscopy, Electrochim. Acta 53 (2008) 7360.

[86] P.K. Shukla, M.E. Orazem, O.D. Crisalle, Validation of the measurement model concept for error structure identification, Electrochim. Acta 49 (2004) 2881.

[87] X.Z. Yuan, C. Song, H. Wang, J. Zhang, Electrochemical impedance spectroscopy in PEM fuel cells. Fundamentals and applications, Springer, London, 2010.

[88] P.R Bevington, D.K. Robinson, Data reduction and error analysis, McGraw-Hill, New York, 2003.

[89] M.A. Tanner, Tools for statistical inference: methods for the exploration of posterior distributions and likelihood functions, Springer, New York, 1996.

[90] I. Herraiz-Cardona, E.M. Ortega, L. Vazquez-Gomez, V. Perez-Herranz, Electrochemical characterization of a NiCo/Zn cathode for hydrogen generation, Int. J. Hydrogen Energ. 36 (2011) 11578. 
[91] I. Herraiz-Cardona, E.M. Ortega, V. Perez-Herranz, Assessment of the roughness factor and the intrinsic catalytic activity for hydrogen evolution reaction on $\mathrm{Ni}$-based electrodeposits, Int. J. Hydrogen Energ. 36 (2011) 9428.

[92] I. Herraiz-Cardona, E.M. Ortega, V. Perez-Herranz, Impedance study of hydrogen evolution on $\mathrm{Ni} / \mathrm{Zn}$ and $\mathrm{Ni}-\mathrm{Co} / \mathrm{Zn}$ stainless steel based electrodeposits, Electrochim. Acta 56 (2011) 1308.

[93] I. Herraiz-Cardona, Desarrollo de nuevos materiales de electrodo para la obtención de hidrogeno a partir de la electrolisis alcalina del agua, Universitat Politècnica de València, Spain, PhD Thesis, 2012.

[94] J. Garcia-Anton, A. Igual-Muños, J.L. Guiñon, V. Perez-Herranz, Horizontal cell for electro-optical analysis of electrochemical processes, P-2000002526 ES patent, 2000.

[95] J.J. Giner-Sanz, E.M. Ortega, V. Pérez-Herranz, Optimization of the electrochemical impedance spectroscopy measurement parameters for PEM fuel cell spectrum determination, Electrochim. Acta 174 (2015) 1290.

[96] A.T. Tran, F. Huet, K. Ngo, P. Rousseau, Artefacts in electrochemical impedance measurement in electrolytic solutions due to the reference electrode, Electrochim. Acta 56 (2011) 8034.

[97] A. Battistel, M. Fan, J. Stojadinovic, F. La Mantia, Analysis and mitigation of the artefacts in electrochemical impedance spectroscopy due to three-electrode geometry, Electrochim. Acta 135 (2014) 133.

[98] J.J. Giner-Sanz, E.M. Ortega, V. Perez-Herranz, Total harmonic distortion based method for linearity assessment in electrochemical systems in the context of EIS, Electrochim. Acta 186 (2015) 598.

[99] B. Hirschorn, M.E. Orazem, On the sensitivity of the Kramers-Kronig relations to nonlinear effects in impedance measurements, J. Electrochem. Soc. 156 (2009) C345. 


\section{LIST OF TABLES.}

Table 1. EIS measurement parameters

Table 2. Experiment order 


\section{LIST OF FIGURES.}

Figure 1. Voigt's circuit

Figure 2. Experimental setup

Figure 3. Change in the Nyquist plot of the system with the perturbation amplitude

Figure 4. Experimental impedance spectrum (real part plot (a) and imaginary part plot (b)) obtained for a perturbation amplitude of $2 \mathrm{~mA}$; and the consistency region built with the described methodology for a confidence level of $95.45 \%$ and 100000 iterations of the Montecarlo algorithm

Figure 5. Experimental impedance spectrum (real part plot (a) and imaginary part plot (b)) obtained for a perturbation amplitude of $3 \mathrm{~mA}$; and the consistency region built with the described methodology for a confidence level of $95.45 \%$ and 100000 iterations of the Montecarlo algorithm

Figure 6. Experimental impedance spectrum (real part plot (a) and imaginary part plot (b)) obtained for a perturbation amplitude of $7 \mathrm{~mA}$; and the consistency region built with the described methodology for a confidence level of $95.45 \%$ and 100000 iterations of the Montecarlo algorithm

Figure 7. Experimental impedance spectrum (real part plot (a) and imaginary part plot (b)) obtained for a perturbation amplitude of $8 \mathrm{~mA}$; and the consistency region built with the described methodology for a confidence level of $95.45 \%$ and 100000 iterations of the Montecarlo algorithm

Figure 8. Experimental impedance spectrum (real part plot (a) and imaginary part plot (b)) obtained for a perturbation amplitude of $9 \mathrm{~mA}$; and the consistency region built with the described methodology for a confidence level of $95.45 \%$ and 100000 iterations of the Montecarlo algorithm

Figure 9. Experimental impedance spectrum (real part plot (a) and imaginary part plot (b)) obtained for a perturbation amplitude of $10 \mathrm{~mA}$; and the consistency region built with the described methodology for a confidence level of $95.45 \%$ and 100000 iterations of the Montecarlo algorithm 
Table 1. EIS measurement parameters

\begin{tabular}{|c|c|}
\hline Measurement parameter & Value \\
\hline Integration time & $1.0 \mathrm{~s}$ \\
\hline Number of integration cycles & 1 cycle \\
\hline Number of stabilization cycles & 10 cycles \\
\hline Maximum stabilization time & $3.0 \mathrm{~s}$ \\
\hline Minimum stabilization cycle fraction & 0.00 \\
\hline
\end{tabular}


Table 2. Experiment order

\begin{tabular}{|c|c|c|c|c|c|c|c|c|}
\hline Order & Block & Experiment & Order & Block & Experiment & Order & Block & Experiment \\
\hline 1 & \multirow{9}{*}{1} & $5 \mathrm{~mA}$ & 10 & \multirow{9}{*}{2} & $3 \mathrm{~mA}$ & 19 & \multirow{9}{*}{3} & $9 \mathrm{~mA}$ \\
\hline 2 & & $4 \mathrm{~mA}$ & 11 & & $9 \mathrm{~mA}$ & 20 & & $7 \mathrm{~mA}$ \\
\hline 3 & & $7 \mathrm{~mA}$ & 12 & & $2 \mathrm{~mA}$ & 21 & & $4 \mathrm{~mA}$ \\
\hline 4 & & $3 \mathrm{~mA}$ & 13 & & $5 \mathrm{~mA}$ & 22 & & $2 \mathrm{~mA}$ \\
\hline 5 & & $2 \mathrm{~mA}$ & 14 & & $10 \mathrm{~mA}$ & 23 & & $6 \mathrm{~mA}$ \\
\hline 6 & & $6 \mathrm{~mA}$ & 15 & & $7 \mathrm{~mA}$ & 24 & & $10 \mathrm{~mA}$ \\
\hline 7 & & $8 \mathrm{~mA}$ & 16 & & $8 \mathrm{~mA}$ & 25 & & $8 \mathrm{~mA}$ \\
\hline 8 & & $9 \mathrm{~mA}$ & 17 & & $4 \mathrm{~mA}$ & 26 & & $5 \mathrm{~mA}$ \\
\hline 9 & & $10 \mathrm{~mA}$ & 18 & & $6 \mathrm{~mA}$ & 27 & & $3 \mathrm{~mA}$ \\
\hline
\end{tabular}




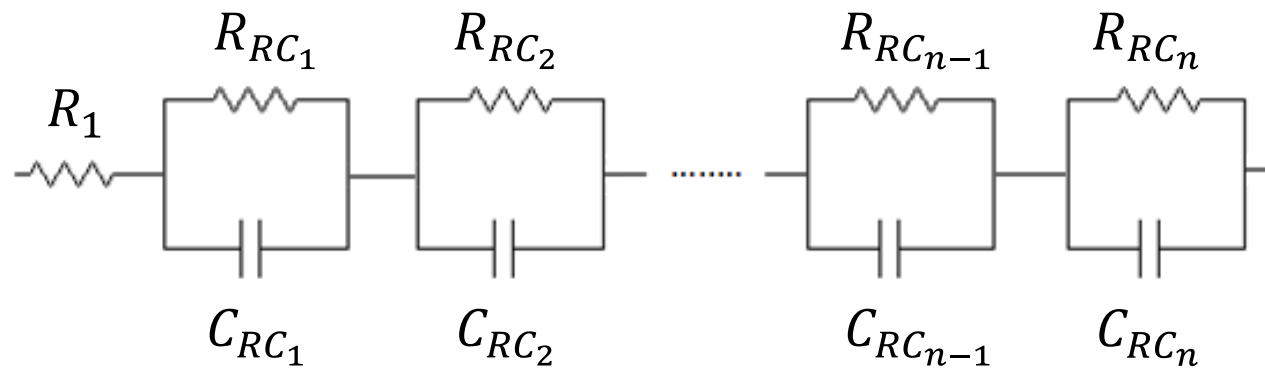

Figure 1. Voigt's circuit 


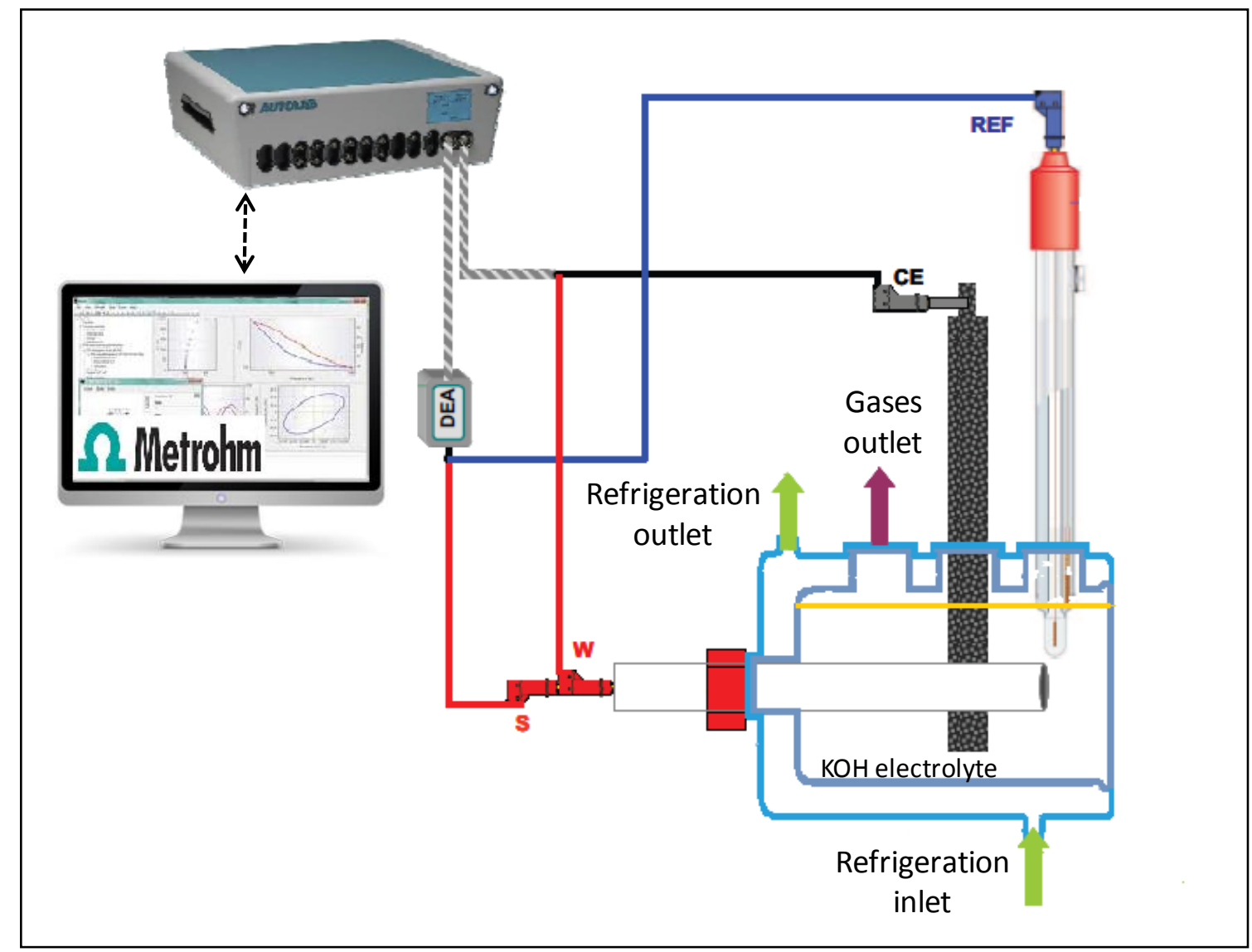

Figure 2. Experimental setup 


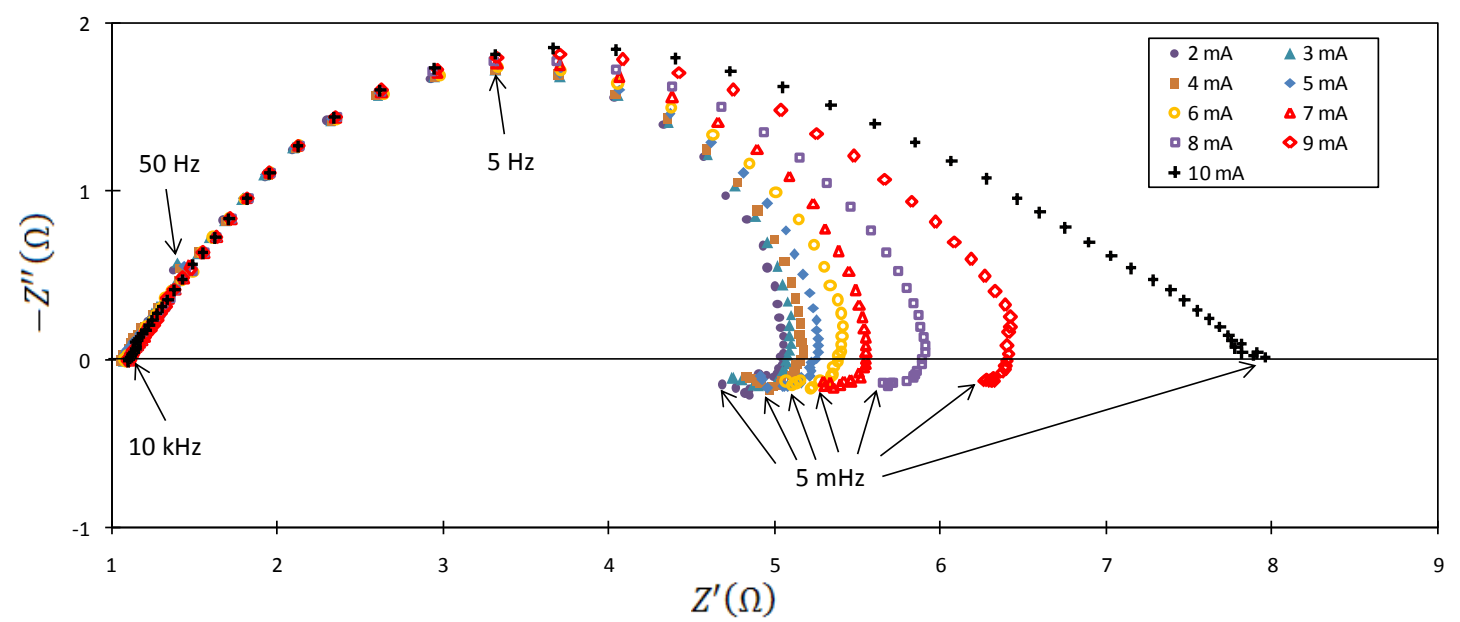

Figure 3. Change in the Nyquist plot of the system with the perturbation amplitude 

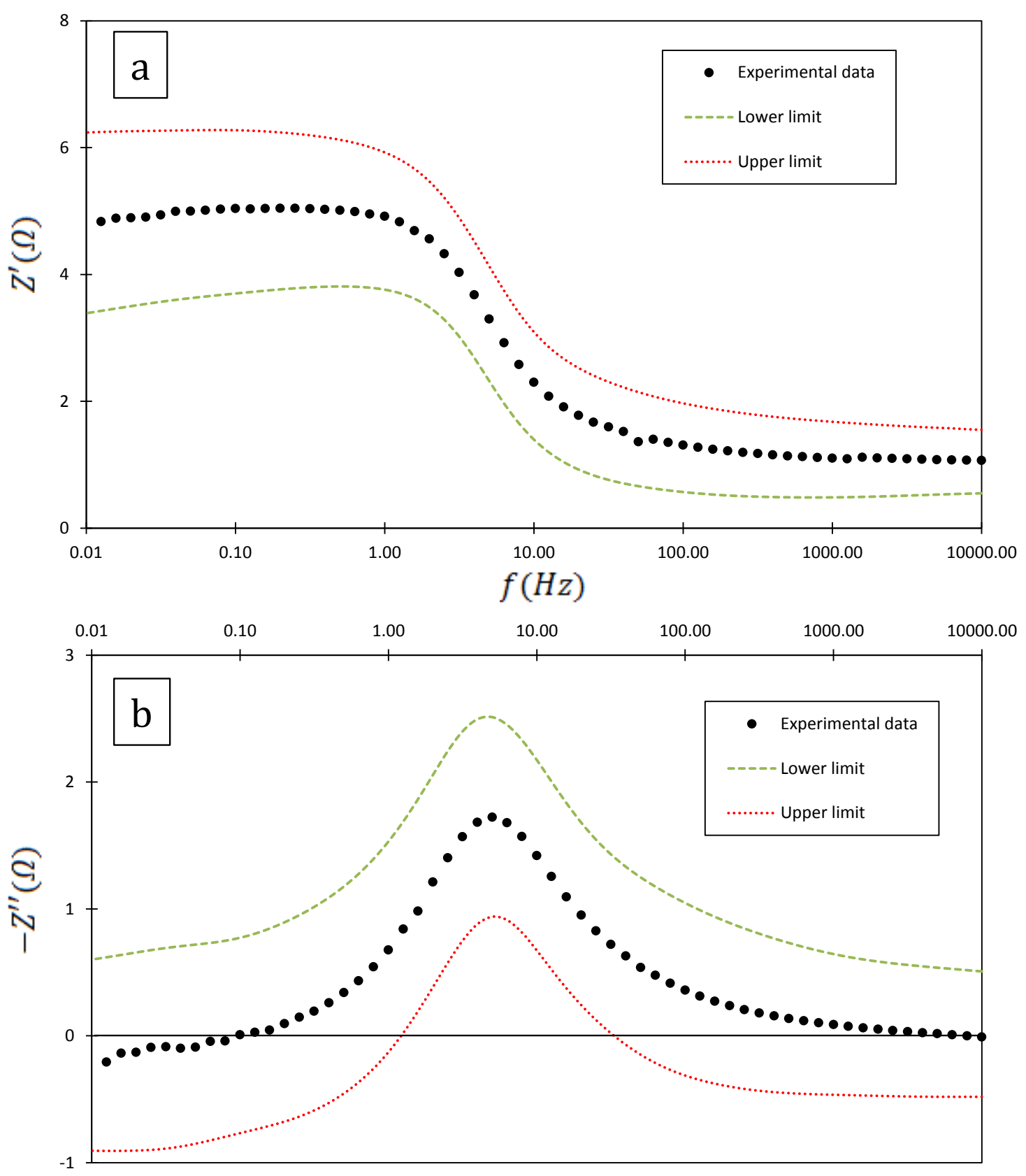

Figure 4. Experimental impedance spectrum (real part plot (a) and imaginary part plot (b)) obtained for a perturbation amplitude of $2 \mathrm{~mA}$; and the consistency region built with the described methodology for a confidence level of $95.45 \%$ and 100000 iterations of the Montecarlo algorithm 

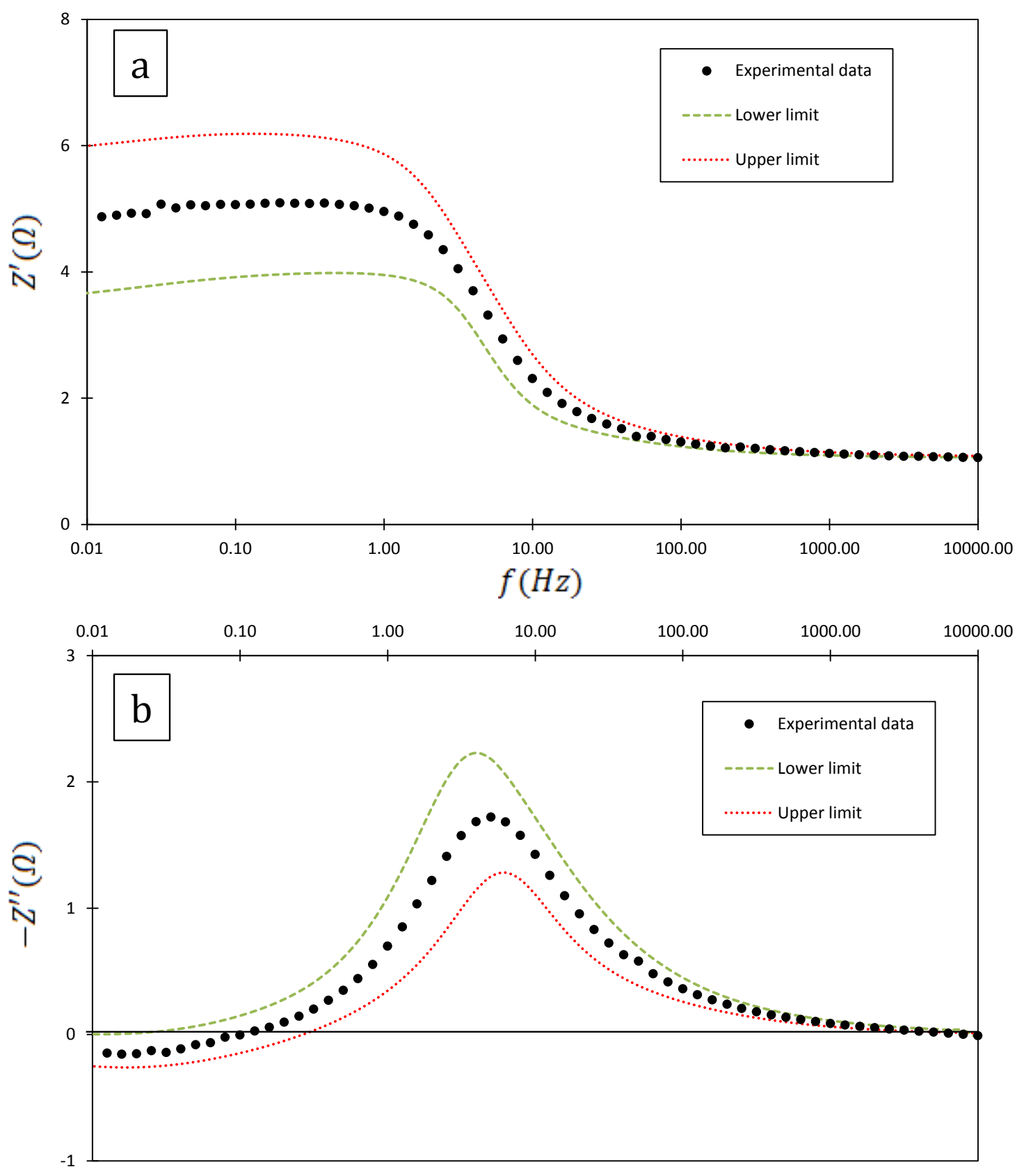

Figure 5. Experimental impedance spectrum (real part plot (a) and imaginary part plot (b)) obtained for a perturbation amplitude of $3 \mathrm{~mA}$; and the consistency region built with the described methodology for a confidence level of $95.45 \%$ and 100000 iterations of the Montecarlo algorithm 

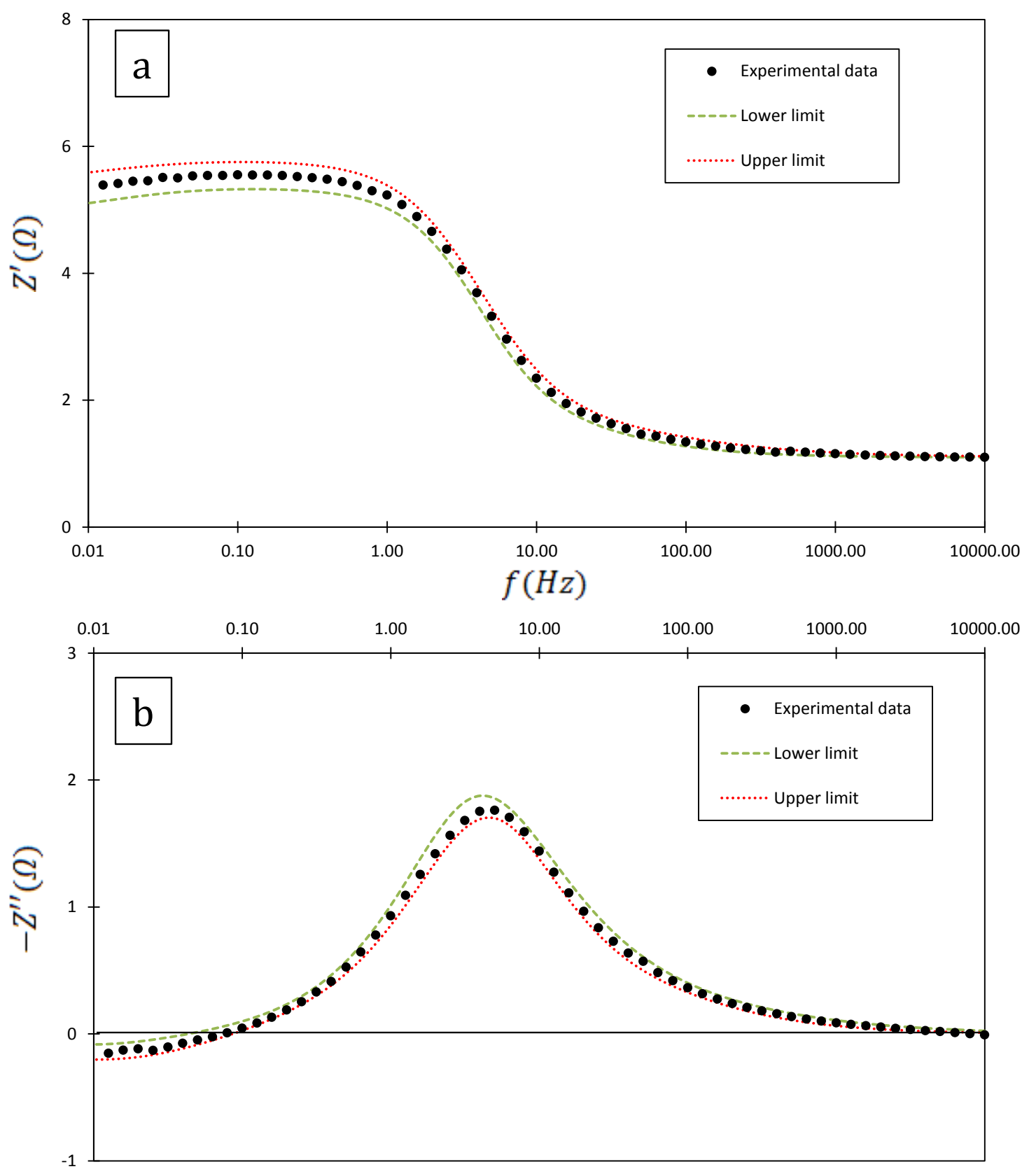

Figure 6. Experimental impedance spectrum (real part plot (a) and imaginary part plot (b)) obtained for a perturbation amplitude of $7 \mathrm{~mA}$; and the consistency region built with the described methodology for a confidence level of $95.45 \%$ and 100000 iterations of the Montecarlo algorithm 

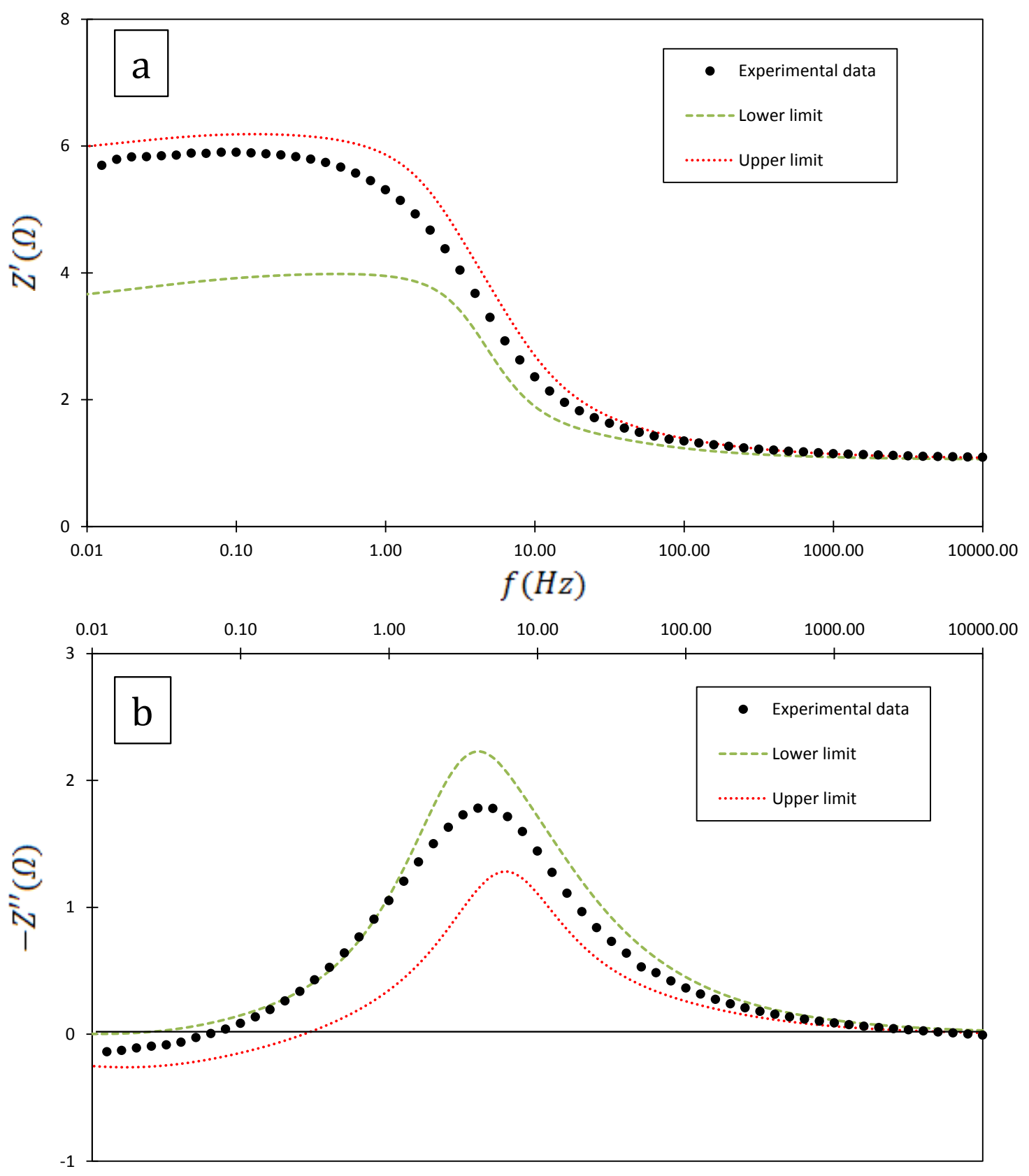

Figure 7. Experimental impedance spectrum (real part plot (a) and imaginary part plot (b)) obtained for a perturbation amplitude of $8 \mathrm{~mA}$; and the consistency region built with the described methodology for a confidence level of $95.45 \%$ and 100000 iterations of the Montecarlo algorithm 

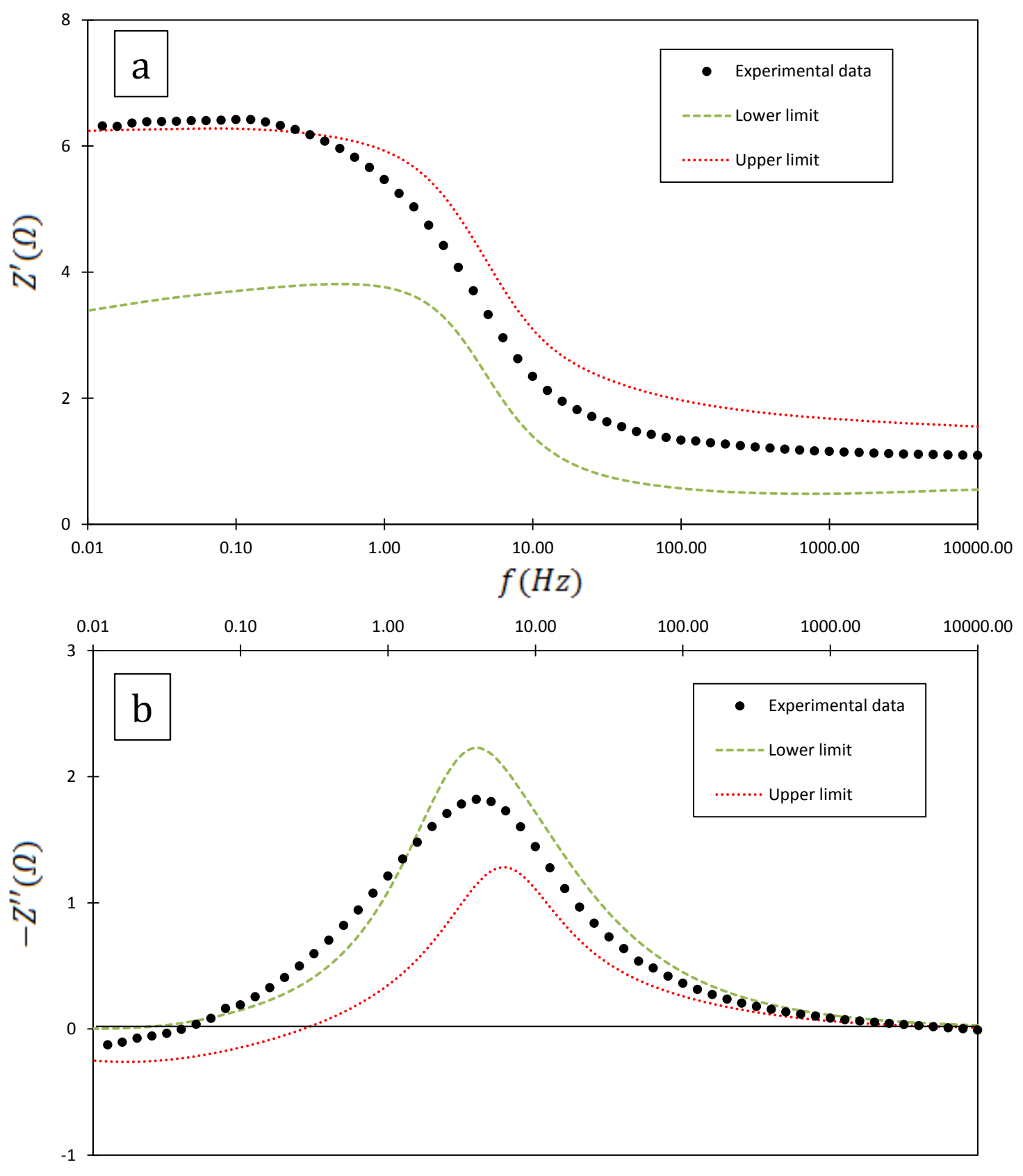

Figure 8. Experimental impedance spectrum (real part plot (a) and imaginary part plot (b)) obtained for a perturbation amplitude of $9 \mathrm{~mA}$; and the consistency region built with the described methodology for a confidence level of $95.45 \%$ and 100000 iterations of the Montecarlo algorithm 

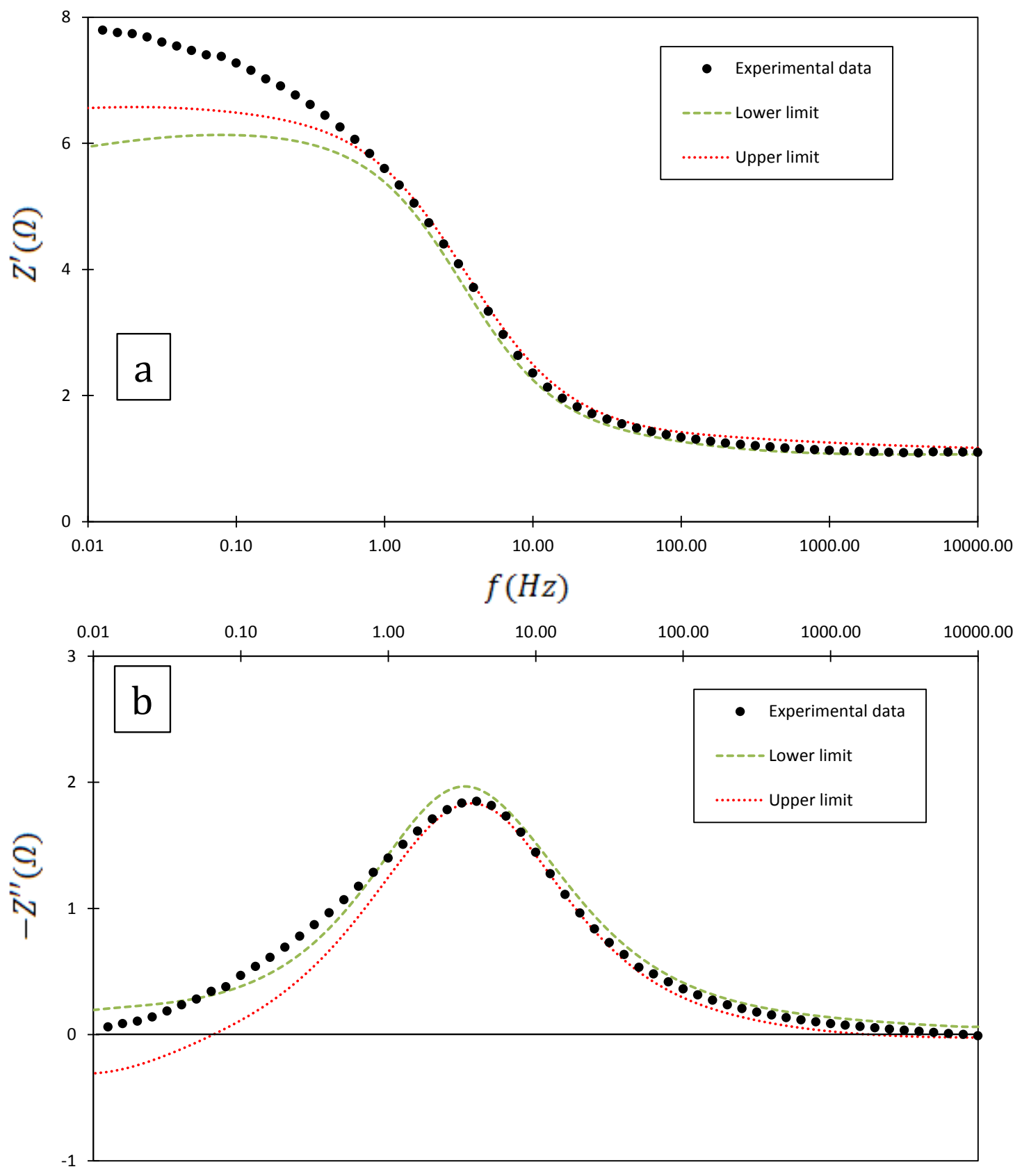

Figure 9. Experimental impedance spectrum (real part plot (a) and imaginary part plot (b)) obtained for a perturbation amplitude of $10 \mathrm{~mA}$; and the consistency region built with the described methodology for a confidence level of $95.45 \%$ and 100000 iterations of the Montecarlo algorithm 